\title{
Heat Transfer by Radiation through Porous Insulations
}

\section{BERT K. LARKIN and STUART W. CHURCHILL}

The University of Michigan, Ann Arbor, Michigan

\begin{abstract}
Radiant transfer through fibrous and foamed insulating materials was investigated theoretically and experimentally. Transmission measurements were made under isothermal conditions with a black-body source varying from $200^{\circ}$ to $800^{\circ} \mathrm{F}$. Bulk density and fiber and pore size were also varied. These data were interpreted successfully in terms of a simple theoretical model. The results provide design information and define the contributions of the several mechanisms of transfer.
\end{abstract}

Because of savings in both space and weight, low-density insulating materials are being used increasingly in consumer appliances, clothing, homes, automobiles, aircraft, and industrial process equipment. The lightweight insulations possess a relatively large amount of void space; usually more than $95 \%$ of their volume is occupied by gas. Typical solid materials now being employed in these insulations are polystyrene, polyurethane, wood fibers, and glass. The insulations can be classified in two types from a geometrical point of view. In one type the solid is the continuous medium, the void space being dispersed bubbles; the other type consists of a matrix of solid fibers held together by a suitable bonding agent. Both types are considered in this investigation. (The particular insulations were chosen for experimental purposes, and direct comparison of their radiant properties is not appropriate.

Heat transfer problems arising from the application of these materials have generally been solved by treating the heat transfer process as if it were wholly a conduction process. On the basis of a conduction mechanism the thermal conductivity should decrease continually with decreasing bulk density, finally approaching the conductivity of the gas filling the void space. The apparent conductivity is actually observed first to decrease and then to increase with decreasing bulk density. This suggests that the other heat transfer processes, such as radiation and free convection, must be contributing appreciably.

Bosworth (2) and Jakob (10) proposed on the basis of different analyses that radiation in porous materials be treated in terms of a radiant conductivity proportional to the cube of the absolute temperature. Topper (17) proposed the use of a radiant heat transfer coefficient with ${ }^{*}$ this same temperature dependence. Verschoor and Greebler (18) derived an expression for the heat transferred by fiber absorption and reradiation in the form of an infinite series which in the

Bert K. Larkin is with The Ohio Oil Co, Fesearch Center, Littleton, Colorado. limit converges to essentially the same expression as that proposed by Jakob. Hamaker (8) presented an earlier and more extensive theoretical analysis of simultaneous radiation and conduction than any of the above. This investigation utilizes a similar mathematical treatment. From measurements of the total rate of heat transfer only, McIntire and Kennedy (13) estimated the radiant contribution to be $25 \%$ in pure Styrofoam. They also found from infrared transmission measurements and from heat transfer tests that the radiant contribution could be reduced by additives with higher reflectivities and absorptivities than polystyrene. Allcut (1) measured the apparent conductivity of glass wool faced first with paper and then with aluminum foil. From these data it can be inferred that radiation comprised from 1 to $10 \%$ of the heat transfer rate.

From infrared transmission measurements (not given) Verschoor and Greebler (18) determined the absorptivity of glass fibers. Radiation rates computed from these data were found to compare favorably with experimental rates of heat transfer in evacuated insulations.

Despite the work indicated above, the effects of the variables that influence radiation are almost wholly undefined, and so the objective of this investigation was to provide quantitative information on these variables. The problem is first formulated in terms of a simple model, in which the parameters are interpreted theoretically and evaluated experimentally. The significance of the experimental results is then discussed, and their application in the prediction of the performance of insulation is illustrated.

\section{MATHEMATICAL REPRESENTATION OF RADIATION}

Radiant transfer through a porous insulation occurs by direct transmission through the "holes," by scattering and by absorption and reradiation. Scattering occurs when electromagnetic waves encounter a discontinuity in refractive
Experimental results are quite limited. index. For fibrous materials this occurs at the surface of the fibers and for foamed materials at the surface of the bubbles. Absorption occurs primarily in transmission through the solid material.

Insofar as the insulation can be treated as an isotropic and continuous material, radiant transfer through porous or dispersed material can be described quite exactly in terms of an integrodifferential equation called the transport equation and a differential energy balance relating the rates of radiation, conduction, and convection. However the transport equation has been integrated only for highly restricted conditions (4) and is not directly applicable for prediction of radiant rates or for correlation of experimental data for thermal insulations. The idealization that the scattered and reradiated energy is propagated only in the forward and backward direction along the $x$ axis, as suggested by Schuster (15), utilized by Hamaker (8), and extended by Chu and Churchill (5), is illustrated in Figure 1. This model yields the simpler and more tractable set of differential equations for the radiant flux density in the forward and backward directions:

$$
\begin{aligned}
& \frac{d I_{1}(x)}{d x}=-n\left(B S_{s}+S_{a}\right) \cdot I_{1}(x) \\
& +n \cdot B \cdot S_{s} \cdot I_{2}(x)+n S_{a} \cdot \sigma \cdot T^{4}(x) \\
& -\frac{d I_{2}(x)}{d x}=-n\left(B S_{s}+S_{a}\right) \cdot I_{2}(x) \\
& \quad+n B \cdot S_{s} \cdot I_{1}(x)+n S_{a} \cdot \sigma \cdot T^{4}(x)
\end{aligned}
$$

and

$$
q=-k_{c} \frac{d T(x)}{d x}+I_{1}(x)-I_{2}(x)
$$

Equation (1) states that in traversing the distance $d x$ the radiant flux density in the forward direction is decreased in the amounts $n \cdot B \cdot S_{s} \cdot I_{1} \cdot d x$ owing to back scattering and $n \cdot S_{A} \cdot I_{1} \cdot d x$ owing to absorption; it is increased in the amounts $n \cdot B \cdot S_{s} \cdot I_{2} \cdot d x$ owing to back scattering of the backward radiant flux density and $n \cdot S_{A} \cdot \sigma \cdot T^{4} \cdot d x$ owing to reradiation. Equation (2) is the analogous balance for the backward radiant flux density. Equations (1) and (2) imply that the spectral distribution of the 


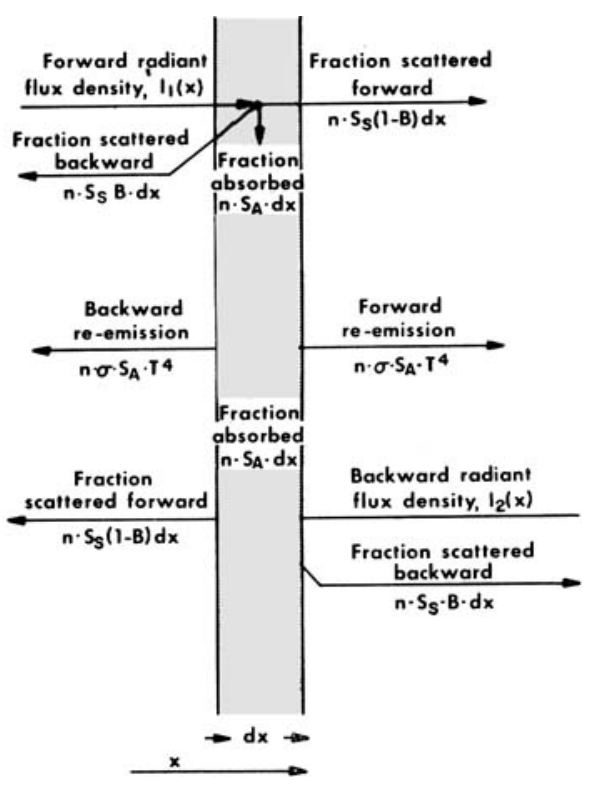

Fig. 1. Two-flux model for radiant transfer.

intercepted and reemitted radiation is the same, a reasonable assumption with the exception of extreme temperature gradients and optically thin slabs. Equation (3) states that the net heat-flux density in the $x$ direction is equal to the rate of conduction plus convection, $-k_{c} d T / d x$, plus the net radiant flux, $I_{1}-I_{2}$.

For convenience the following grouping of parameters will be defined:

$$
\begin{gathered}
M=n\left(B S_{s}+S_{a}\right) \\
N=n B S_{s} \\
P=M-N=n S_{a}
\end{gathered}
$$

$M, N$, and $P$ are the interception, backscattering, and absorption cross sections of a unit volume of insulation and will be assumed to be constant with respect to $x$. Obviously only two of these three parameters are independent, but all three are retained for convenience. Equations (1) and (2) then reduce to

$$
\begin{aligned}
\frac{d I_{1}}{d x} & =-M I_{1}+N I_{2}+P \sigma T^{4} \\
-\frac{d I_{2}}{d x} & =-M I_{2}+N I_{1}+P \sigma T^{4}
\end{aligned}
$$

The boundary conditions for insulation faced with sheets of opaque material consist of the temperatures of the

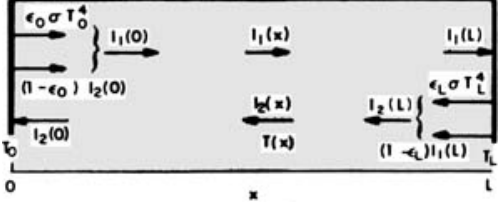

Fig. 2. Boundary conditions for two-flux model and opaque surfaces.

boundary, $T_{0}$ at $x=0$ and $T_{L}$ at $x=L$, and the radiant flux balances

$I_{1}(0)=\sigma T_{0}{ }^{4} \epsilon_{0}+\left(1-\epsilon_{0}\right) I_{2}(0)$

and

$I_{2}(L)=\sigma T_{L}{ }^{4} \epsilon_{L}+\left(1-\epsilon_{0 L}\right) I_{1}(L)$

which state that the radiant flux entering the insulation consists of the radiant emission from the boundary surface plus the fraction of the incident radiation reflected by the bounding surface (Figure 2). Alternative boundary conditions, such as for unfaced insulation, have been considered by Harnaker ( 8 ).

For nonabsorbing insulation $[P=0$, $M=N]$ and constant $k_{c}$, Equations (3), (7), and (8) can be readily solved with the preceding boundary conditions. The temperature varies linearly across the insulation, and the total heat flux is

$$
\begin{aligned}
q= & \frac{k\left(T_{0}-T_{L}\right)}{L} \\
& +\frac{\sigma\left[T_{0}{ }^{4}-1\right.}{\frac{1}{\epsilon_{0}}+\frac{1}{\epsilon_{L}}-1+N L}
\end{aligned}
$$

The first term on the right is the rate of conduction and convection and the second term the rate of radiation. Both are constant across the insulation.

The equations are not readily solvable for absorbing materials $[P \neq 0]$. The three equations can be reduced to a pair of equations in any two of the three variables, but all such pairs are nonlinear. Although particular solutions of these pairs are obtainable from an analogue computer, greater accuracy and usefulness appeared possible with an approximate analytical solution.

If the net radiant heat-flux density is essentially constant or is a small fraction of the total heat transfor rate, and $k_{c}$ is constant, it is apparent from Equation (3) that the temperature gradient will be nearly constant. The assumption of a constant temperature gradient and hence a linear variation in temperature across the insulation linearizes Equations (7) and (8). This linearized pair can be solved by ordinary methods to yield the radiant rate

$$
\begin{aligned}
& q_{1}(x)=I_{1}(x)-I_{2}(x) \\
& =\frac{\left(2-\epsilon_{0}\right)(M-N) W+A Z}{C} \\
& \cdot \sinh \sqrt{M^{2}-N^{2}} x \\
& +\frac{\epsilon_{0} \sqrt{M^{2}-N^{2}} W-G \cdot Z}{C} \\
& \cdot \cosh \sqrt{M^{2}-N^{2}} x+2 X(x)
\end{aligned}
$$

where

$$
\begin{aligned}
& A=\epsilon_{L} \sqrt{M^{2}-N^{2}} \\
& \cdot \sinh \sqrt{M^{2}-N^{2}} L \\
& +\left(2-\epsilon_{L}\right)(M-N) \\
& \cdot \cosh \sqrt{M^{2}-N^{2}} L \\
& W=\epsilon_{L} Y(L)-\left(2-\epsilon_{L}\right) X(L) \\
& Z=\epsilon_{0} Y(0)+\left(2-\epsilon_{0}\right) X(0) \\
& G=\left(2-\epsilon_{L}\right)(M-N) \\
& \cdot \sinh \sqrt{M^{2}-N^{2}} L \\
& +\epsilon_{L} \sqrt{M^{2}-N^{2}} \\
& \cdot \cosh \sqrt{M^{2}-N^{2}} L \\
& C=\left[(M-N)\left(2-\epsilon_{0}-\epsilon_{L}\right)\right. \\
& \left.+M \epsilon_{0} \epsilon_{L}\right] \sinh \sqrt{M^{2}-N^{2}} L_{\text {. }} \\
& +\sqrt{M^{2}}-\overline{N^{2}}\left(\epsilon_{0}+\epsilon_{L}-\epsilon_{0} \epsilon_{L}\right) \\
& \cdot \cosh \sqrt{M^{2}-\overline{N^{2} I}} \\
& X(x)=\frac{4 \sigma\left(T_{0}-T_{L}\right)}{(M+N) L} \\
& \cdot\left[T^{3}(x)+\frac{6\left(T_{0}-T_{L}\right)^{2}}{\left(M^{2}-N^{2}\right) L^{2}} T(x)\right] \\
& Y(x)=\frac{12 \sigma\left(T_{0}-T_{L}\right)^{2}}{\left(M^{2}-N^{2}\right) L^{2}} \\
& \cdot\left[T^{2}(x)+\frac{2\left(T_{n}-T_{L}\right)^{2}}{\left(M^{2}-N^{2}\right) L}\right]
\end{aligned}
$$

For $\sqrt{M^{2}-N^{2} L}>5$, which proved to be the case for an inch or more of all the commercial insulations studied

$$
q_{r}(0) \cong \epsilon_{0}\left\{\frac{\left[(M+N) \epsilon_{L}+\sqrt{M^{2}-N^{2}}\left(2-\epsilon_{L}\right)\right] X(0)}{(M-N)\left(2-\epsilon_{0}-\epsilon_{i}\right)+M \epsilon_{0} \epsilon_{3}}-\frac{\left[\left(2-\epsilon_{L}\right)(M-N)+\epsilon_{L} \sqrt{M^{2}-N^{2}}\right] \cdot Y(0)}{\sqrt{M^{2}-N^{2}}\left(\epsilon_{0}+\epsilon_{L}-\epsilon_{0} \epsilon_{L}\right)}\right\}
$$

If also

$$
\begin{aligned}
& T_{0} \gg \frac{3\left(T_{0}-T_{L}\right)}{(M-N) L} \\
& \quad q_{r}(0) \cong \frac{4 \sigma \epsilon_{0}\left(T_{0}-T_{L}\right)}{(M+N) L}\left\{\frac{(M+N) \epsilon_{L}}{(M-N)\left(2-\epsilon_{0}-\epsilon_{L}\right)+} \frac{\sqrt{M^{2}}-N^{\overline{2}}\left(2-\epsilon_{L}\right)}{\left(M \epsilon_{0} \epsilon_{L}+\sqrt{M^{2}-N^{2}}\left(\epsilon_{0}+\epsilon_{L}-\epsilon_{0} \epsilon_{L}\right)\right.}\right\} T_{0}{ }^{3}
\end{aligned}
$$




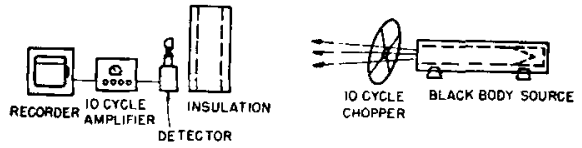

Fig. 3. Schematic diagram of equipment.

\section{THE EVALUATION OF RADIATION PARAMETERS} FROM ELECTROMAGNETIC THEORY

Equations (11) and (12) yield the radiant contribution to heat transfer for nonabsorbing and absorbing insulation in terms of $N$ and $M$ and of $N$ respectively. These parameters, which are functions of the physical properties of the insulations, can in principle be evaluated from electromagnetic theory.

\section{Foamed Insulations}

If the foamed insulation is assumed to contain a random dispersion of uniformly sized spheres of gas, the number of spheres per unit volume is found from a simple volume balance to be related to the sphere size and gas, solid and bulk densities as

$$
n=\frac{3}{4 \pi R^{3}} \cdot \frac{\rho_{S}-\rho}{\rho_{S}-\rho_{G}}
$$

The scattering cross section is usually expressed in terms of a scattering coefficient equal to the ratio of the scattering to the geometric cross section; that is

$$
K_{s}=S_{s} / \pi R^{2}
$$

Hence

$$
N=\frac{3 B K_{s}}{4 R} \cdot \frac{\rho_{S}-\rho}{\rho_{S} \rho_{G}}
$$

The properties $B$ and $K_{s}$ can be com-

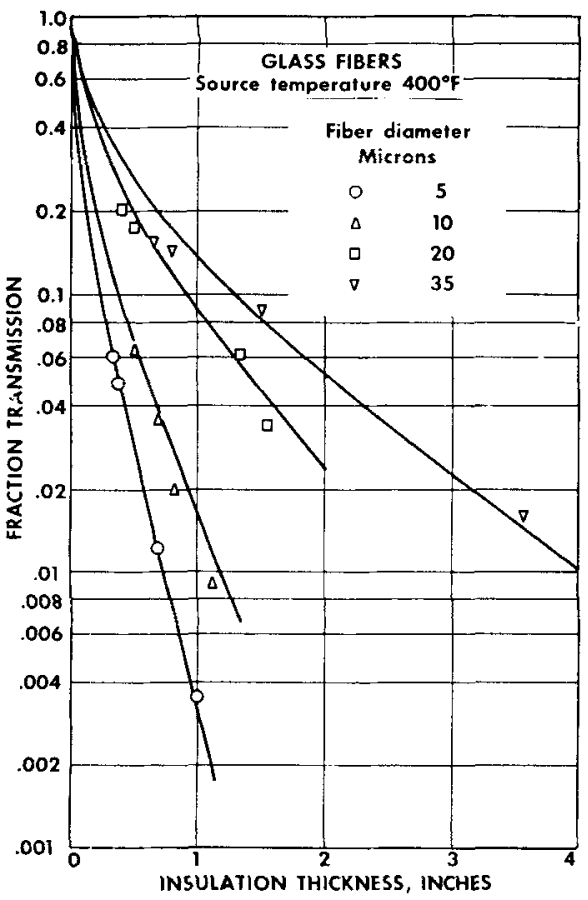

Fig. 5. Transmission of radiation through glass fibers.

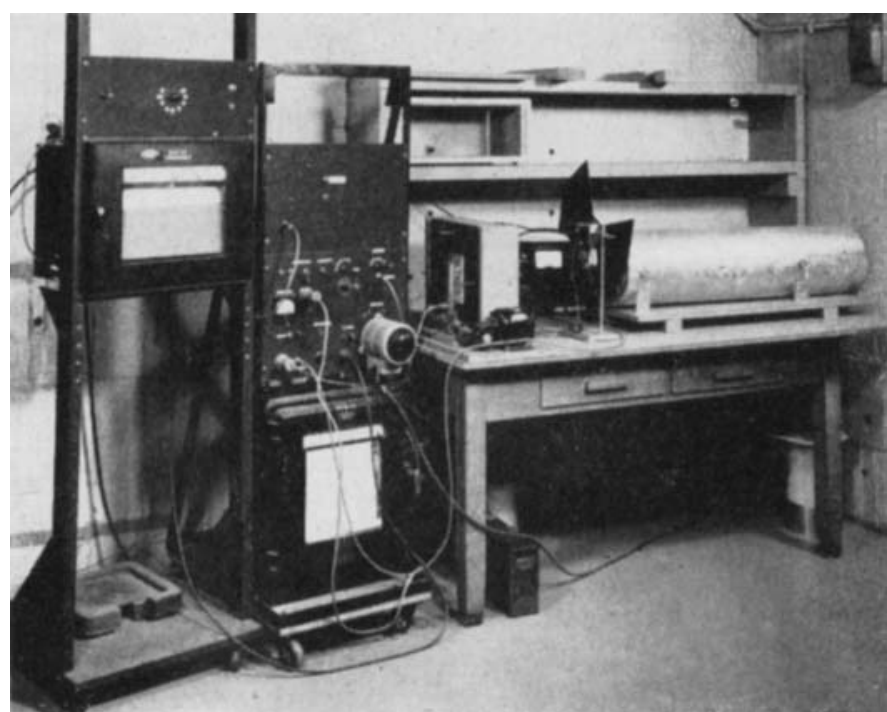

Fig. 4. Experimental apparatus.

puted from the Mie solutions of the Maxwell equations $(14,6,8)$ if the relative index of refraction of the solid and gas is known. Unfortunately the required calculations are extensive, and the index of refraction of insulating materials is not known in the thermal range of wave lengths. However the effect of pore size can be inferred from limiting values of the Mie solution. For pores much larger than the wave length of the radiation, $B K_{s}$ approaches a constant value, and hence $N$ varies inversely with pore size; for pores much smaller than the wave length, $B K_{s}$ is proportional to the fourth power of the pore radius, and hence $N$ is proportional to the radius cubed. $N$ is a measure of the ability of the insulation to scatter radiation backward, and the larger the value of $N$ the smaller the rate of heat transfer by radiation. Since the large-pore approximation indicates that small pores are most effective and the small-pore approximation that large spheres are most effective, an optimum pore size is to be

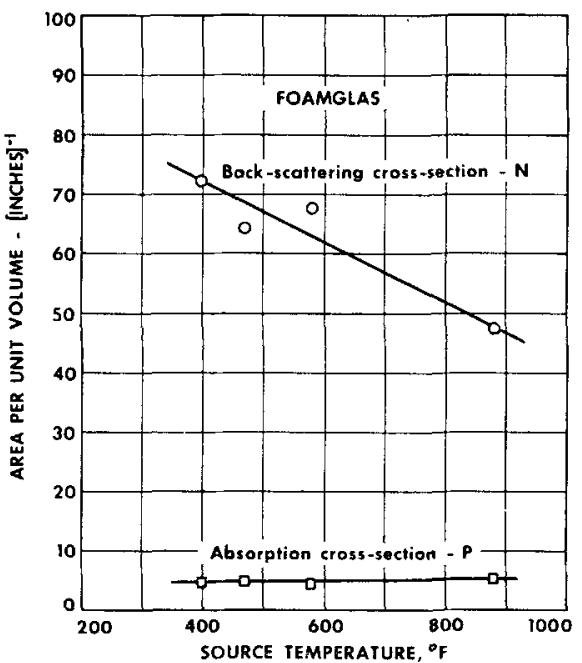

Fig. 6. Radiation parameters for Foamglas. expected for a given porosity if scattering is controlling.

Theoretical evaluation of the absorption cross section is less certain. When one assumes that each pore is associated with a cube of solid which is large with respect to the wave length

$$
S_{a}=n^{-\frac{3}{3}}\left(1-e^{-\beta y}\right)
$$

$y=R\left(\frac{\rho-\rho_{G}}{\rho_{S}-\rho_{G}}\right)\left(\frac{\rho_{S}-\rho_{G}}{\rho_{S}-\rho} \cdot \frac{4 \pi}{3}\right)^{\frac{1}{3}}$

and hence

$P=\frac{1}{R}\left(\frac{\rho_{S}-\rho_{G}}{\rho_{S}-\rho} \cdot \frac{4 \pi}{3}\right)^{\frac{1}{3}}\left(1-e^{-\beta y}\right)$

The necessary information on the absorptivity of insulating materials is currently unavailable. However, for small pores $P$ would appear to be independent of pore size, and for large pores $P$ would appear to be inversely proportional to pore size.

\section{Fibrous Insulations}

For a random matrix of uniformly sized cylinders with axes perpendicular

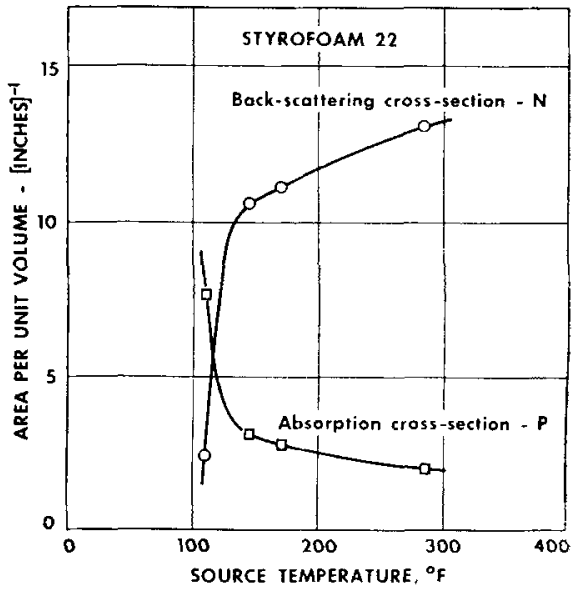

Fig. 7. Radiation parameters for Styrofoam-22. 
to the direction of heat flow the following expressions are analogous to those for foamed insulations:

$$
N=\frac{4 B K_{s}}{\pi D_{f}} \cdot \frac{\rho-\rho_{G}}{\rho_{S}-\rho_{G}}
$$

and

$$
P=\frac{4 K_{a}}{\pi D_{f}} \cdot \frac{\rho-\rho_{G}}{\rho_{S}-\rho_{G}}
$$

$B K_{s}$ and $K_{x}$ can be computed from electromagnetic theory if the index of refraction and absorptivities are known. A summary of the theory and the results of illustrative calculations are presented in (11) and (12). Some of these values computed for specific indices of refraction are subsequently compared with the experimental data. The qualitative behavior of $N$ and $P$ for cylinders is similar to that for spheres. $N$ and $P$ vary inversely with fiber diameter for $D_{f} \gg \lambda$ and directly with fiber diameter squared for $D_{f} \ll \lambda$.

\section{Limitations}

The evaluation of the radiation parameters from electromagnetic theory is limited severely by current information on the index of refraction and absorptivity of the materials and even with these properties involves extensive calculations on a high-speed computer. The present contribution of theory is primarily in the qualitative prediction of effects.

\section{EXPERIMENTAL. EVALUATION OF RADIATION PARAMETERS}

The radiation parameters $N$ and $M$ were evaluated experimentally for representative and specially selected insulations by transmission measurements with a black-body source at a series of temperatures. Reradiation and background radiation were minimized by modulating the source and using a sharply tuned $10 \mathrm{cycle} / \mathrm{sec}$. amplifier. The insulation was maintained at room temperature to minimize conduction and further to minimize reradiation.

A schematic diagram of the equipment is shown in Figure 3 and a photograph of the equipment in Figure 4. The blackbody source consisted of a 36-in.-long by 4-in.-diameter copper tube wrapped with heating wires and covered with insulation. One end of the tube was closed with a plug having an internal conical cavity. Temperatures along the inside of the pipe were maintained uniformly within $\pm 5^{\circ} \mathrm{F}$. by control of the several segments of the heating wire. The chopper used to modulate the source consisted of a two-bladed propeller driven by a synchronous motor. The radiation falling on the insulation varied periodically as a $10 \mathrm{cycle} / \mathrm{sec}$. square wave.

The detector was similar to those employed in infrared spectrometers and contained a blackened thermocouple. It was mounted to traverse a 3 -in. path

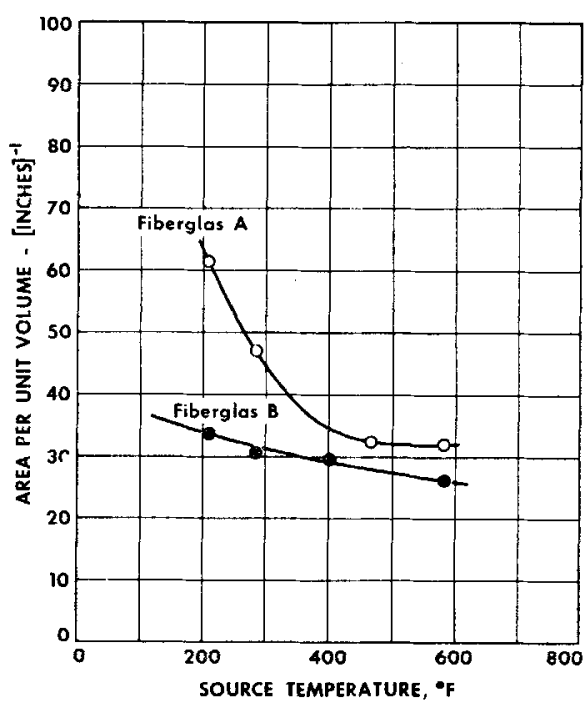

Fig. 8. Back-scattering cross-section $N$ of Fiberglas $A$ and $B$.

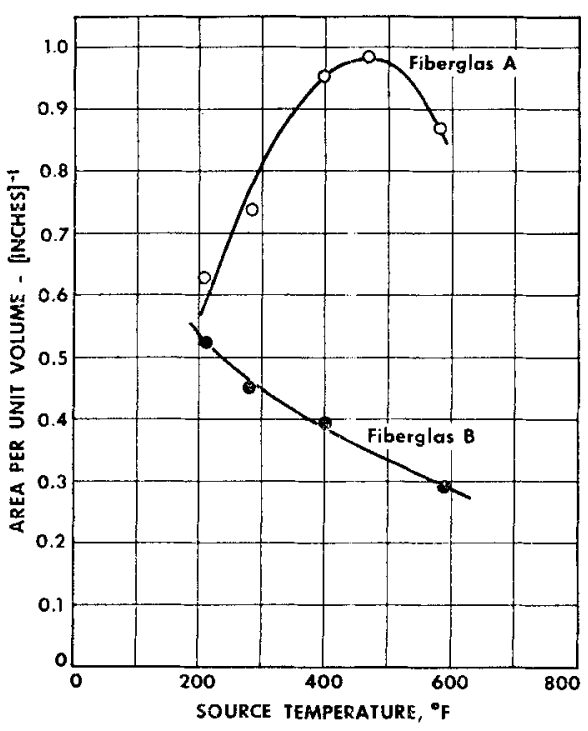

Fig. 9. Absorption cross-section $P$ of Fiberglas $A$ and $B$.

parallel to the surface of the insulation. Since the signal was often as low as a few hundredths of a microvolt, a highgain amplifier was needed. A recorder was utilized to facilitate averaging the signal obtained with nonuniform insulations.

No change in signal was observed when part of the specimen was masked, an indication that an infinite slab was successfully simulated. The signal was observed to vary as the inverse square of the distance from an uncollimated source, indicating that the detector response was linear. The angular response of the detector was also tested and found to be acceptable. The detector signal was found to be closely proportional to the difference of the fourth power of the

$$
I_{1}(L)=\frac{1}{\cosh L \sqrt{M^{2}-N^{2}}+\frac{M}{\sqrt{M^{2}-N^{2}}} \cdot \sinh L \sqrt{M^{2}-\overline{N^{2}}}}
$$

temperature of the source and receiver, indicating that both the source and receiver were nearly "black" or equally "gray." Additional details concerning the equipment are available 11 .

Several hours were required for the source to attain a steady state. The unattenuated radiation was first measured with the detector. From this trace and prior measurements of the variation of the flux with distance from the source the flux incident upon the insulation was computed. A traverse was then made with the specimen in place, and the amplifier gain increased as necessary. The average of this flux divided by the average flux incident upon the specimen was defined as the fractional transmission. If the electrical and thermal noise was appreciable, an additional traverse was made with the source shielded, and this signal was subtracted from both the unattenuated and attenuated readings. (The original data are tabulated in 11.)

Table 1 gives the properties of the tested insulations. The specimens of commercial insulation were 11 -in. squares and varied from $1 / 8$ to $1 \mathrm{in}$. in thickness. The specimens of unbonded glass fibers were cylinders 6 in. in diameter and less than $1 / 2$ in. in thickness. The mass, area, and thickness of the thickest specimens were measured and the bulk density computed. The thinner specimens were assumed to have the same bulk density, and their thickness was calculated from the mass and area. In all of the fibrous insulations the fibers were oriented perpendicular to the heat flux.

\section{INTERPRETATION OF TRANSMISSION MEASUREMENTS}

In terms of the components of the intensity previously defined, the flux incident upon the insulation is $I_{1}(0)$, and the transmitted flux is $I_{1}(L)$. Since the detector and insulation were at room temperature, and since the detector responded only to $10 \mathrm{cycle} / \mathrm{sec}$. energy, reradiation from the fibers and surroundings was neglected. Equations (7) and (8) then reduce to

$$
\begin{aligned}
\frac{d I_{1}}{d x} & =-M I_{1}+N I_{2} \\
-\frac{d I_{2}}{d x} & =-M I_{2}+N I_{1}
\end{aligned}
$$

and the boundary conditions can be written

$$
I_{1}(0)=1
$$

and

$$
I_{2}(L)=0
$$

The transmission is then 
Expanding Equation (27) in series one gets

$$
\begin{aligned}
I_{\mathrm{I}}(L) & =\frac{2 \sqrt{M^{2}-N^{2}} e^{-2 L \sqrt{M^{2}-N^{2}}}}{M+\sqrt{M^{2}-N^{2}}} \\
& \cdot \sum_{n=0}^{\infty}(-1)^{n+1} \\
& \cdot\left[\frac{M-\sqrt{M^{2}-N^{2}}}{M+\sqrt{M^{2}-N^{2}}}\right]^{n} \\
& \cdot e^{-2 n I \sqrt{M^{2}-N^{2}}}
\end{aligned}
$$

For $L \sqrt{M^{2}-\overline{N^{2}}}>5$, the series in Equation (28) approaches unity. Under such conditions a semilog plot of transmission vs. thickness should yield a straight line. $M$ and $N$ could then be determined from the slope and intercept of the least square line through the data. A typical set of data is shown in Figure 5. It is evident that the logarithm of the transmission varies nearly linearly with large thickness in Figure 5 but must have curvature throughout to approach unity at zero thickness. Therefore the constants $M$ and $N$ were evaluated by successive approximation. First $M$ and $N$ were estimated from the straight-line region of the data. The series portion of Equation (28) was next evaluated by the use of the approximate values of $M$ and $N$. The two groups of constants in the balance of Equation (28) were then evaluated by least squares, and $M$ and $N$ were calculated. This process was repeated as necessary. The curves shown in Figure 5 were obtained in this way.

\section{Commercial Insulations}

The experimentally determined values of the radiation parameters for four commercial insulations are plotted vs. source temperature in Figures 6 to 9 . Since scattering and absorption depend primarily on the spectral distribution of the incident radiation rather than on the temperature of the material, these data are believed to be representative for insulations at these temperatures. As noted previously $N$ and $P$ are the cross sections per unit volume for back scattering and absorption respectively. The larger the values of $N$ and $P$, the more effective is the insulation in reducing radiant transfer.

For the Foamglas $N$ is much larger than $P$, an indication that scattering is predominant over absorption. The decrease in $N$ with temperature is difficult to interpret without data on the index of refraction. $P$ appears to be relatively independent of temperature, possibly owing to the absorptivity of the two major constituents, glass and carbon. Glass absorbs little in the visible range of wave lengths, moderately in the near infrared, and strongly in the far infrared. Carbon absorbs strongly in the visible but is relatively transparent in the infrared. As the temperature increases, the radiant energy shifts to shorter wave lengths, and the absorption may shift from glass to carbon.

For Styrofoam-22 $N$ increases and $P$ decreases with temperature. At $100^{\circ} \mathrm{F}$.

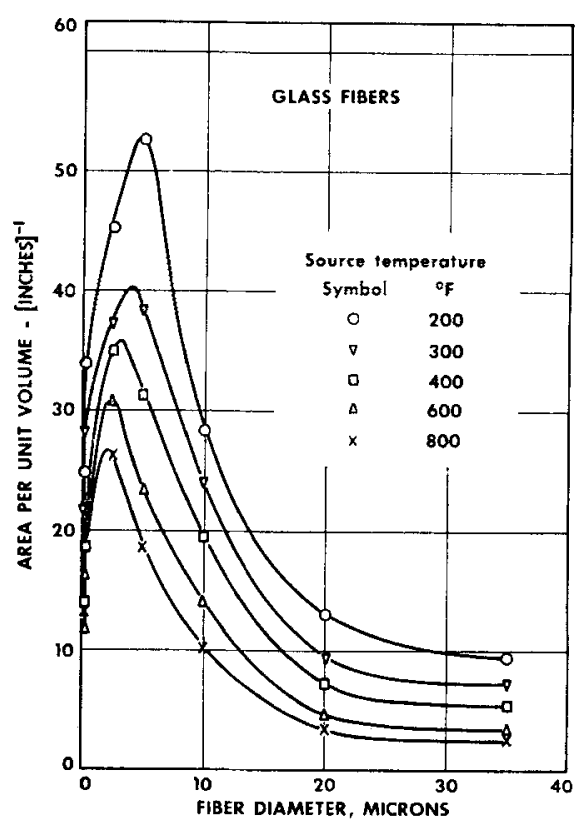

Fig. 10. Back-scattering cross-section $N$ of glass fibers.

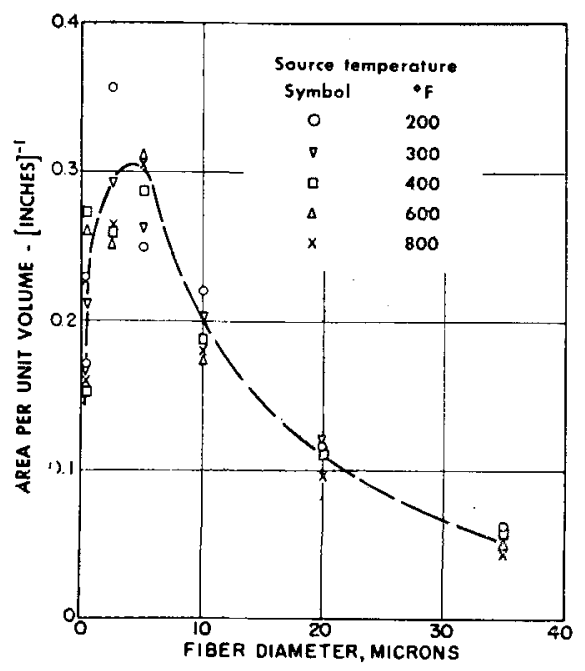

Fig. 11. Absorption cross-section $P$ of glass fibers.

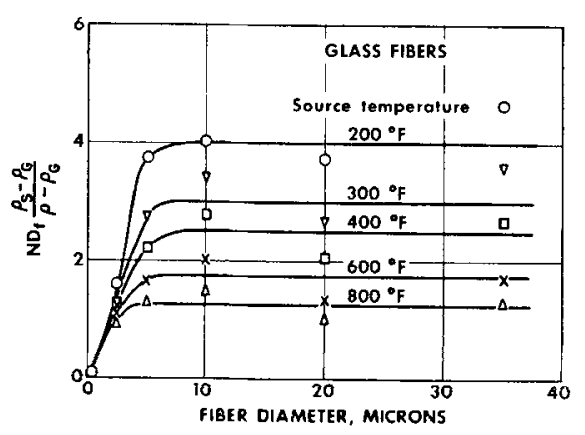

Fig. 12. Dimensionless scattering parameter for glass fibers. absorption is predominant, but above $150^{\circ} \mathrm{F}$. back scattering predominates. Since the absorptivity of polystyrene is qualitatively similar to that of glass, the temperature dependence of $P$ is understandable.

For the Fiberglas insulations absorption is negligible with respect to back scattering. The maximum in $P$ for Fiberglas $A$ is probably attributable to the bonding agent, which is $20 \mathrm{wt} . \%$ of the insulation, rather than to the glass.

\section{Unbonded Glass Fibers}

The values of $N$ and $P$ obtained with unbonded, uniformly sized glass fibers are shown in Figures 10 and 11 . The fluence of fiber size on scattering is very great and demonstrates the maximum predicted by theory. The maximum shifts from about $5 \mu$ at $200^{\circ} \mathrm{F}$. to about $2.5 \mu$ at $800^{\circ} \mathrm{F}$; $P$ demonstrates similar behavior. The values of $N$ and $P$ are replotted in Figures 12 and 13 in the form which theory suggests should minimize dependence on fiber size for large fibers. The deviations due to experimental error are emphasized in this plot. Insufficient data were obtained to test the corresponding asymptotic relationships for small fibers.

Since the index of refraction and absorptivity of the glass fibers are not known as functions of wave length, the integration

$$
\begin{array}{r}
B K_{s}\left(T, D_{f}, m\right) \\
=\frac{\int_{0}^{\infty} B_{\lambda} K_{s \lambda} E_{\lambda T} d \lambda}{\sigma T^{4}}
\end{array}
$$

was carried out with the use of theoretical values of $B_{\lambda} K_{s \lambda}(11,12)$ for no absorption and fixed indices of 1.5, 2.0, and 2.5 (Figure 14). Qualitative agreement with the experimental data is demonstrated. This agreement is most significant, since it supports the theoretical basis for the correlation of the data and suggests that rafiant transfer through insulations can be predicted quantitatively on the basis of the electromagnetic theory from measurements of the index of refraction and the absorptivity of the solid material only. However it should not be inferred from Figure 14 that the index of refraction of the fibers lies between 1.5 and 2.0 , since the imaginary part of the index of refraction of glass is not zero, as assumed for the calculations.

\section{COMPUTATION OF RADIANT TRANSFER}

Equations (11) and (12) and the data in Figures 8 through 13 permit prediction of the rate of heat transfer by radiation through porous materials of the types tested for any fiber size, bulk density, temperatures, or boundary emissivities. These predictions will be illustrated 
in terms of the radiant conductivity, defined as

$$
\begin{aligned}
k_{r}=\frac{I_{1}-I_{2}}{\frac{-d T}{d x}} & =\frac{q}{\frac{-d T}{d x}} \\
& -k_{c}=k-k_{c}
\end{aligned}
$$

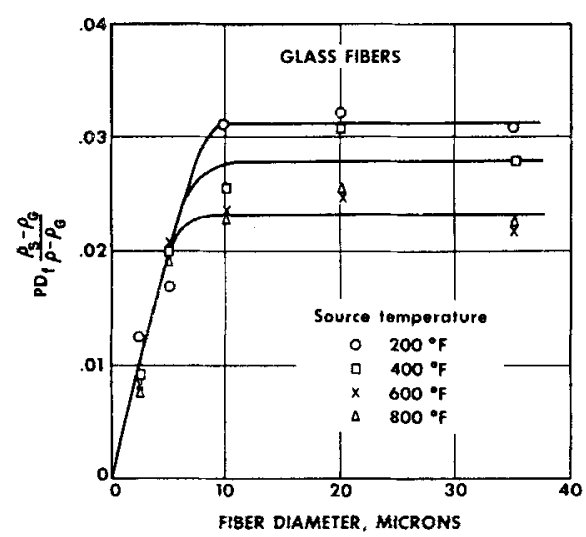

Fig. 13. Dimensionless absorption parameter for glass fibers.

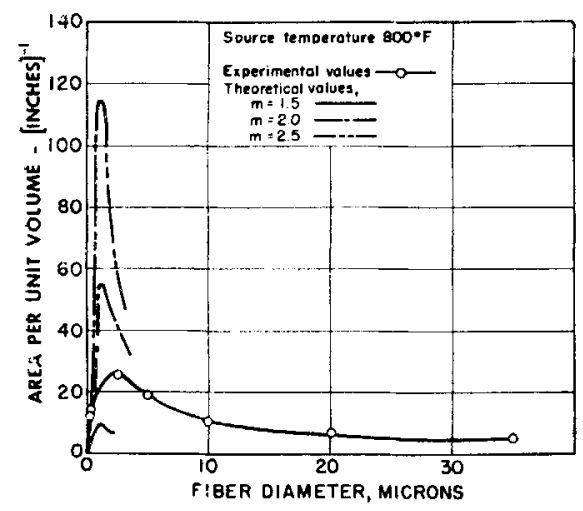

Fig. 14. Comparison of theoretically and experimentally evaluated parameters for glass fibers.

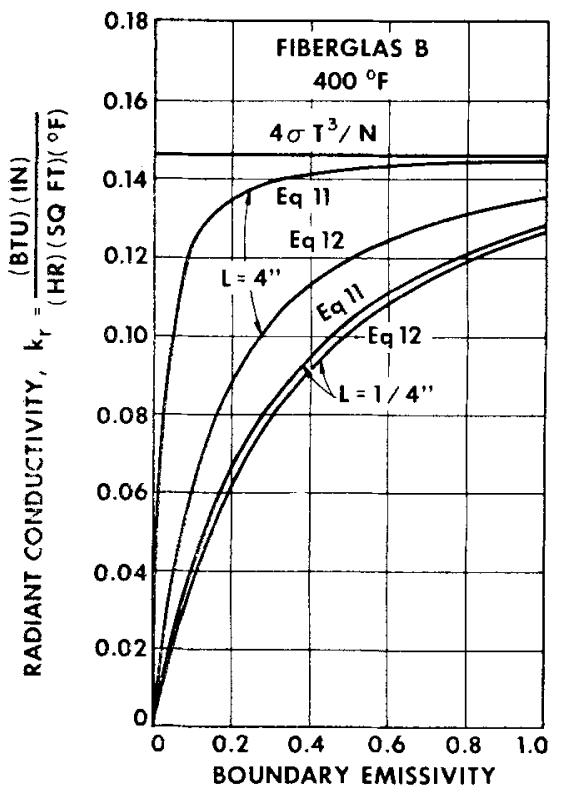

Fig. 15. Effect of boundary emissivity on radiant conductivity of Fiberglas $B$. and in terms of the fraction of the heat transferred by radiation, $k_{r} / k$.

The absorption and scattering properties of materials are critically dependent upon the spectrum of the incident radiation but are essentially independent of the temperature of the material itself (10). Hence the data of this investigation, which were obtained with different source temperatures but with the material at room temperature, were correlated in terms of the source temperature. However these properties can be interpreted and applied in terms of the material temperature at any point in an insulation when the source of radiation is the surrounding material at essentially the same temperature. For simplieity the succeeding illustrative figures were prepared for a vanishingly small temperature difference and hence constant $k_{r}$ and $k_{r}$. Similar plots taking into account the variation of $k_{c}$ and $k_{r}$ with temperature could readily be prepared for any specific temperature difference.

For all the materials investigated, except Styrofoam-22 below $150^{\circ} \mathrm{F}$., absorption is sufficiently small so that Equation (11) is a fair approximation. It indicates that the effects of temperatures, boundary emissivities, fiber or pore size, and bulk density upon the radiant conductivity are dependent on the thickness of the insulation. However for thick slabs such that

$$
\begin{aligned}
& L \gg\left(\frac{1}{\epsilon_{0}}+\frac{1}{\epsilon_{L}}-1\right) / N \\
k_{r}= & \frac{\sigma\left[T_{0}^{2}+T_{L}^{2}\right]\left[T_{0}+T_{L}\right] L}{\frac{1}{\epsilon_{0}}+\frac{1}{\epsilon_{L}}-1+N L} \\
\rightarrow & \left.\frac{\sigma\left[T_{0}{ }^{2}+T_{L}^{2}\right]\left[T_{0}\right.}{N}+T_{L}\right] \\
\cong & \frac{4 \sigma T^{3}}{N}
\end{aligned}
$$

Thus insofar as Equation (31) is a valid representation, the totil heat transfer process in insulation can be treated as a conduction process with an effective conductivity that depends on temperature and the structure of the insulation but is independent of thickness and boundary conditions. If $\left[\left(1 / \epsilon_{0}\right)+\left(1 / \epsilon_{L}\right)-1\right] \gg N L$, the radiant conductivity of a nonabsorbing insulation depends critically upon the boundary emissivitirs and the thickness. If absorption is dominant $(M \gg N)$, Equation (14) suggests that

$$
k_{r}(0) \cong \frac{4 \sigma}{M} \frac{\epsilon_{0} T^{3}}{M}
$$

The dependence of the radiant conductivity of Fiberglas $B$ (at the boundary) on thickness and boundary emissitivity is indicated in Figure 15. The

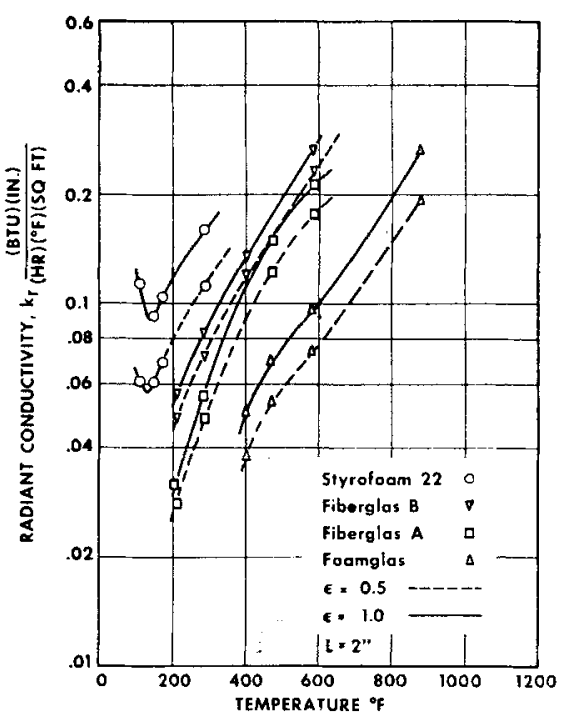

Fig. 16. Effect of temperature on radiant conductivity.

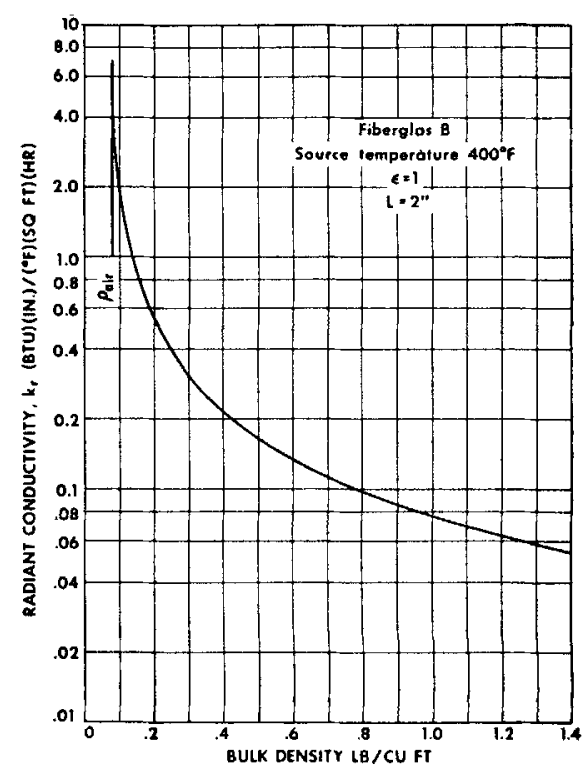

Fig. 17. Effect of bulk density on radiant conductivity of Fiberglas $B$.

limitations of the approximations represented by Equations (11) and (31) are also indicated.

Figure 16 illustrates the influence of temperature level on radiant transfer. Owing to the decrease in the radiation parameters with temperature, $k_{r}$ is proportional to the second power or less of the absolute temperature rather than the third power predicted by earlier work. The importance of boundary emissivity is again apparent.

The effect of bulk density on radiant transfer through a fibrous insulation is illustrated in Figure 17. The curve terminates at a density of $0.07 \mathrm{lb}$./cu. ft., corresponding to no fibers, and a radiant conductivity corresponding to exchange between two infinite plates. The effect 


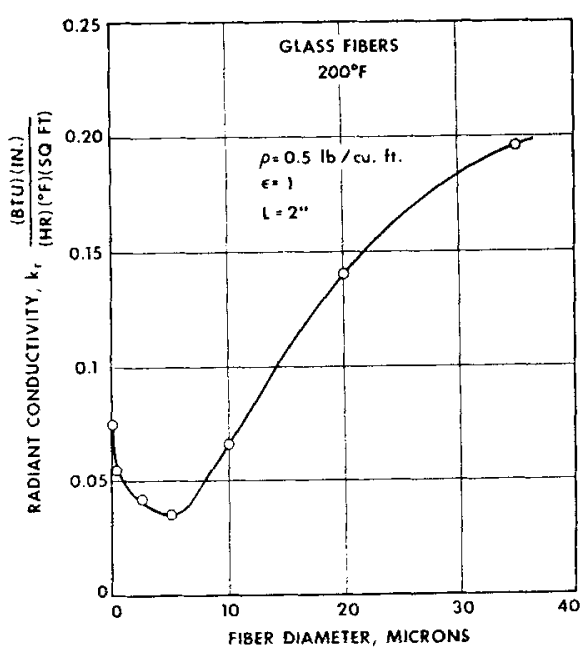

Fig. 18. Effect of fiber size on radiant conductivity of glass fibers.

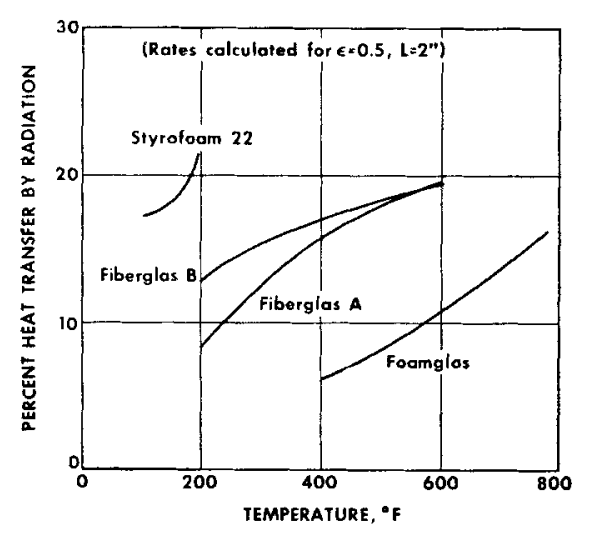

Fig. 19. Radiant contribution to heat transfer in insulations. on foamed insulations is more complicated. If the bulk density is increased for a fixed bubble size, $P$ increases and $N$ decreases. The net effect depends upon the relative importance of scattering and absorption.

Figure 18 shows the variation of the radiant conductivity with fiber size. The optimum fiber size for the reduction of radiation is the same here as that which maximizes $N$, since absorption is negligible.

'The fraction of the heat transfer due to radiation is plotted in Figure 19 for the commercial insulations. A boundary emissivity of 0.5 was arbitrarily assumed for these computations. A higher value of $\epsilon$ would lead to a greater radiant contribution. The total conductivities with which the radient conductivities are compared are given in Table 1 . The computed rates of radiation are considerably less than those estimated by Verschoor and Greebler (18) from heat transfer data for evacuated fibrous insulations but are in relatively good agreement with the estimates of McIntire and Kennedy (13) for foamed insulations. Figure 19 should not be construed as a comparison of the merits of the three types of insulation. Other insulations of the same types could undoubtedly be chosen to reverse the relative magnitudes of the radiant conductivities.

\section{SUMMARY AND CONCLUSIONS}

A simple model has been developed for radiant transfer through porous insulating materials. The two parameters in this model can in principle be evaluated from electromagnetic wave theory, but

Table 1. Physical Properties of Insulations Studied

\begin{tabular}{|c|c|c|c|c|c|c|}
\hline Insulation & Manufacturer & $\begin{array}{l}\text { Refer- } \\
\text { ence }\end{array}$ & $\begin{array}{l}\text { Mean } \\
\text { fiber or } \\
\text { pore } \\
\text { size, } \mu\end{array}$ & $\begin{array}{c}\text { Bulk } \\
\text { density, } \\
\text { lb./cu.ft. }\end{array}$ & $\begin{array}{l}\text { Temper } \\
\text { ature, } \\
{ }^{\circ} \mathrm{F} \text {. }\end{array}$ & $\begin{array}{c}k^{*}, \\
\text { B.t.u./ } \\
\text { (hr.) (sq. ft.) } \\
\left({ }^{\circ} \text { F. } / \text { in. }\right)\end{array}$ \\
\hline Styrofoam-22 & Dow Chemical & 16 & 0.05 (in.) & 1.8 & $\begin{array}{r}50 \\
100 \\
150\end{array}$ & $\begin{array}{l}0.26 \\
0.295 \\
0.335\end{array}$ \\
\hline Foamglas & $\begin{array}{r}\text { Pittsburgh } \\
\text { Corning }\end{array}$ & 7 & 0.05 (in.) & 9.0 & $\begin{array}{l}100 \\
200 \\
300 \\
400 \\
500\end{array}$ & $\begin{array}{l}0.43 \\
0.50 \\
0.55 \\
0.62 \\
0.62\end{array}$ \\
\hline Fiberglas $A$ & $\begin{array}{l}\text { Owens Corning } \\
\text { Fiberglas }\end{array}$ & 9 & 1.0 & 0.6 & $\begin{array}{l}100 \\
200 \\
300 \\
400\end{array}$ & $\begin{array}{l}0.24 \\
0.32 \\
0.42 \\
0.58\end{array}$ \\
\hline Fiberglas $B$ & $\begin{array}{l}\text { Owens Corning } \\
\text { Fiberglas }\end{array}$ & 9 & 3.0 & 0.6 & $\begin{array}{l}100 \\
200 \\
300 \\
400\end{array}$ & $\begin{array}{l}0.31 \\
0.43 \\
0.595 \\
0.83\end{array}$ \\
\hline $\begin{array}{l}\text { Unbonded } \\
\text { glass fibers }\end{array}$ & $\begin{array}{l}\text { Owens Corning } \\
\text { Fiberglas }\end{array}$ & 9 & $\begin{array}{c}0.17 \\
0.20 \\
2.5 \\
5.0 \\
10.0 \\
20.0 \\
35.0\end{array}$ & $\begin{array}{l}0.50 \\
0.50 \\
0.50 \\
0.50 \\
0.50 \\
0.50 \\
0.50\end{array}$ & & \\
\hline
\end{tabular}

the monochromatic index of refraction and absorptivity which is required is not currently available for the materials used in insulations. Illustrative calculations for arbitrary indices of refraction are in qualitative agreement with values obtained from black-body transmission measurements. The experimental values of the parameters permit calculation of radiant transfer for a wide range of conditions and materials.

Radiation contributes 5 to $20 \%$ of the heat transfer through typical insulations of Fiberglas, Foamglas, and Styrofoam. Scattering is the primary mechanism for the restriction of radiation in Fiberglas and Foamglas, but either scattering or absorption may be controlling in Styrofoam depending on the temperature. Consequently increasing the bulk density decreases the radiant transfer through Fiberglas and Foamglas but may increase or decrease the transfer through Styrofoam. In view of the small absorption by all the insulations tested, the addition of strongly absorbing materials should be effective.

The radiant conductivity is proportional to the second power or less of the absolute temperature for all the materials tested. Calculations indicate that the emissivity of the material bounding the insulation may be important under some circumstances. An optimum diameter in the range from 2 to $5 \mu$ exists for the restriction of radiation through glass fibers, and an optimum pore size is presumed to exist for foamed materials.

\section{ACKNOWLEDGMENT}

K. F. Gordon, R. L. Hess, C. W. Peters, and J. L. York of The University of Michigan and Marion Hollingsworth, Jr., of the Owens Corning Fiberglas Corporation provided invaluable advice. J. $\mathrm{H}$. Chin, George C. Clark, and Phillip H. Scott assisted directly in the work. Insulations were provided by the Dow Chemical Company and the Pittsburgh Corning Corporation and special materials as well as insulations by the Owens Corning Fiberglas Corporation. Financial support was provided B. K. Larkin by the E. I. du Pont Fellowship in Chemical Engineering.

\section{NOTATION}

$B=$ fraction of scattered radiation scattered into the background hemisphere.

$D_{f}=$ fiber diameter

$E_{T}=$ emission function

$I_{1}(x)=$ radiant flux density in the forward (positive $x$ ) direction

$I_{2}(x)=$ radiant flux density in the backward (negative $x$ ) direction

$K_{a}=$ absorption coefficient of a fiber or pore; ratio of absorbed to geometrically obstructed radiation

$K$. = scattering coefficient of a fiber 
or pore; ratio of scattered to geometrically obstructed radiation

$k=$ effective total conductivity

$k_{\text {r }} \quad=$ effective conductivity for gaseous conduction, solid conduction, and natural convection only

$k_{r} \quad=$ equivalent conductivity for radiation

$L=$ thickness of insulation

$M=$ interception cross section per unit volume of insulation

$m \quad=$ index of refraction

$N=$ back scattering cross section per unit volume of insulation

$n=$ number of scatters per unit volume

$P \quad=$ absorption cross section per unit volume of insulation

$q=$ total heat-flux density (a constant across insulation)

$q,(x)=$ net radiant heat-flux density

$R=$ pore radius

$S_{a}=$ absorption (and emission) cross section per scatter

$S_{s}=$ scattering cross section per scatter

$T(x)=$ absolute temperature

$x=$ distance through insulation

$y=$ mean thickness of solid associated with a pore

\section{Greek Letters}

$\beta=$ exponential absorption coeffcient of solid niaterial

$\epsilon \quad=$ emissivity of boundary surface of insulation

$\lambda=$ wave length

$\rho \quad=$ bulk density

$\rho_{G}=$ gas density

$\rho_{S} \quad=$ solid density

$\sigma \quad=$ Stefan Boltzm: $\sigma n$ constant

\section{Subscripts}

$0-$ at $x=0$ boundary

$L-$ at $x=L$ boundary

$\lambda$ - monochromatic

\section{LITERATURE CITED}

1. Alleut, E. A., Inst. Mech. Engrs. London, 232 (1951).

2. Bosworth, R. L. C., "Heat Transfer Phenomena," John Wiley, New York (1952).

3. Chu, C. M., G. C. Clark, and S. W. Churchill, "Angular Distribution Coefficients," Univ. Michigan Press, Ann Arbor, Michigan (1957).

4. Chu, C. M., and S. W. Churchill, Trans. Inst. Radio Engrs., AP-4, 2, 142 (1956).

5. — J. Phys. Chem., 59, 855, (1955).

6. Clark, G. C., C. M. Chu, and S. W.
Churchill, J. Opt. Soc. Am., 47, 81, (1957).

7. "Foamglas Insulation for Piping and Process Equipment," Pittsburgh Corning Corp., Pittsburgh, Pennsylvania (1951).

8. Hamaker, H. C., Phillips Research Repts., 2, 55, 103 (1947).

9. Hollingsworth, Marion, Jr., Owens Corning Fiberglas Corp., Newark, Ohio, private communication.

10. Jakob, Max, "Heat Transfer," Vol. I, John Wiley, New York (1949).

11. Larkin, B. K., Ph.D. thesis, Univ. Michigan, Ann Arbor, Michigan (1957).

12. —, and S. W. Churchill, J. Opt. Soc. $A m ., 49,188$ (1959).

13. McIntire, O. R., and R. N. Kennedy, Chem. Eng. Progr., 44, 727 (1948).

14. Mie, Gustav, Ann. Physik, 25, 377 (1908).

15. Schuster, Arthur, Astrophys. J., 21, 1 (1905).

16. "Styrofoam," The Dow Chemical Company, Midland, Michigan (1955).

17. Topper, Leonard, Ind. Eng. Chem., 47, 1377 (1955).

18. Verschoor, J. D., and Paul Greebler, Trans., Am. Soc. Mech. Engrs. 74, 961 (1952).

Manuscript reccived May 21, 1958; revision re. ceived November 24, 1958; paper accepted November 28,1958 . Paper presented at A.I.Ch.E. Montreal meeting.

\section{The Economic Design of Mixer-Settler Extractors}

ROBERT E. TREYBAL

New York University, New York, New York

Relationships are developed for establishing the most economic values of the major variables of a liquid-extraction process, including the concentrations of solute in recycled solvent and rejected raffinate, the solvent-to-feed ratio, and for mixer-settlers certain of the design features. Consideration is given to costs of extraction and solvent recovery, as well as to the value of unextracted solute and lost solvent. For the design of mixer settlers scale-up relationships are developed to permit prediction of the stage efficiency of a large extractor from experimental data taken on a small scale. These are expressed in terms of a scale-up index relating the relative size of the mixer with the volumetric rates of liquid flow. It is shown that the cost of a multistage extractor increases with scale up in a different fashion from the stage efficiency, depending upon the scale-up index used. A detailed study of the costs for a typical case led to the development of economic scale-up indexes which, because they cover nearly a fivefold ratio of fixed to operating costs, are of fairly general utility. The common practice of scaling up with equal holding times on the large and small scale is shown to be amply safe from the point of view of stage efficiency to be realized on the large scale but usually uneconomic.

Simplification of the complete system of equations permits rapid estimation of the most economic circumstances for any type of countercurrent extractor of which the cost per stage is proportional to $Q^{a}$.

Despite the facts that mixer settlers are probably the oldest form of commercial liquid extractors and that new countercurrent tower devices are invented every year, mixer settlers have never been wholly superseded. In recent years there has evon been an increased tendency to use them. The many reasons for this need not be dwelt upon here. It is sufficient to note that it is generally believed that small-scale experiments provide a reliable guide to the design of large-scale equipment.

Although there have been a number of new mixer-settler designs in the past few 